http://dx.doi.org/10.35381/r.k.v6i12.1371

\title{
Parámetros productivos en la incubación de huevos considerados como no aptos procedentes de reproductoras pesadas
}

\section{Productive parameters in the incubation of eggs considered unsuitable from broiler breeders}

\author{
Junior Stalin Vargas-Hidalgo \\ junior.vargas@espoch.edu.ec \\ Escuela Superior Politécnica de Chimborazo, Riobamba \\ Ecuador \\ https://orcid.org/0000-0003-1245-7609 \\ Diego Armando Masaquiza-Moposita \\ dmasaquiza@espoch.edu.ec \\ Escuela Superior Politécnica de Chimborazo, Riobamba \\ Ecuador. \\ https://orcid.org/0000-0001-5176-8261 \\ Nelson Rene Ortiz-Naveda \\ nelson.ortiz@espoch.edu.ec \\ Escuela Superior Politécnica de Chimborazo, Riobamba \\ Ecuador. \\ https://orcid.org/0000-0003-1083-8475
}

Recepción: 15 de marzo 2021

Revisado: 15 de mayo 2021

Aprobación: 15 de junio 2021

Publicación: 01 de julio 2021 


\title{
RESUMEN
}

La investigación tiene por objetivo analizar los parámetros productivos en la incubación de huevos considerados como no aptos (por su peso y forma) procedentes de reproductoras pesadas. Se evaluó el efecto de la incubación de huevos considerados como no aptos (pequeños, grandes y deformes) procedentes de reproductoras pesadas, para ser comparados con un grupo control (huevos aptos o normales). Los índices de incubabilidad estos variaron numéricamente entre 79.48 y $89.28 \%$, con mejores respuestas en los huevos normales, sin embargo, se encontró que el tamaño y forma de los huevos no influye en este parámetro. Los índices de viabilidad alcanzados fueron de 83.19 a $91.05 \%$, que pertenecen a los obtenidos en los huevos pequeños y normales, respectivamente, pero que estadísticamente son iguales.

Descriptores: Producción alimentaria; industria alimentaria; agroindustria. (Palabras tomadas del Tesauro UNESCO).

\begin{abstract}
The research aims to analyze the productive parameters in the incubation of eggs considered unsuitable (due to their weight and shape) from broiler breeders. The effect of the incubation of eggs considered as unfit (small, large and deformed) from broiler breeders was evaluated, to be compared with a control group (suitable or normal eggs). The hatchability indices these ranged numerically between 79.48 and $89.28 \%$, with better responses in normal eggs, however, it was found that the size and shape of the eggs did not influence this parameter. The viability indices reached were from 83.19 to $91.05 \%$, which belong to those obtained in small and normal eggs, respectively, but which are statistically equal.
\end{abstract}

Descriptors: Food production; food industry; agroindustry. (Words taken from the UNESCO Thesaurus). 


\section{INTRODUCCIÓN}

La incubación es el acto por el que los animales ovíparos (sobre todo las aves) empollan o incuban los huevos sentándose sobre ellos para mantenerlos calientes y así se puedan desarrollar los embriones. (De Marchi et al. 2008). En los actuales modelos de explotación de reproductoras pesadas, se ha eliminado la incubación natural. Desde una perspectiva económico-productiva, una incubación natural tendría un impacto negativo, dado que el ave interrumpiría durante esta fase su puesta y disminuiría con ello el número de huevos puestos por ciclo, que es precisamente el objetivo primario de producción, es conveniente, por tanto, recurrir a la incubación artificial.

En este sentido, el huevo presenta requerimientos básicos incluyen la necesidad de proteínas, vitaminas y minerales y constituye un alimento recomendable para todas las edades, muy adecuado en las etapas de crecimiento y necesidades fisiológicas especiales (embarazo y lactancia) y en la dieta de personas mayores, por lo tanto, el huevo constituye aproximadamente el $30 \%$ de la proteína animal que se consume en el mundo y es evidente, que las aves serán la principal fuente de carne en el próximo siglo (López-Sobaler et al. 2017).

Esta industria ha enfrentado muchos obstáculos en el pasado, pero el futuro ciertamente presentará desafíos y oportunidades aún mayores. Una de las fuerzas significativas de la industria de pollos de ceba, ha sido su avidez para adoptar nuevas tecnologías; quizás más que cualquier otra área del sector agropecuario, ha estado dispuesta a probar y adoptar nuevas ideas, para que el productor de esta línea en el siglo XXI continúe siendo competitivo (Mendoza-Rodríguez et al. 2016).

Se ha optado por nuevos métodos y equipos que reducen la mano de obra, aumentan la eficiencia, mejoran y refuerzan consistentemente los rendimientos productivos, esto los lleva a ser aún más importantes que en el pasado. Estos aspectos que se describen para la producción de pollos de ceba pueden extrapolarse a la producción de huevos con el 
fin de mejorar la eficiencia en la explotación de este propósito (Cortes-Cuevas et al. 2016).

Es reconocido que uno de los elementos en que los avicultores coinciden son los resultados que no sólo se logran con un buen trabajo en las etapas finales (ceba y ponedoras comerciales) que son fiables, hay que lograr reemplazos en cantidad y calidad que aseguren las necesidades de estas producciones terminales. Es por ello que, en la actualidad, gastan grandes recursos y esfuerzos en etapas intermedias, como son la incubación artificial y los reproductores, donde se obtienen los animales para las crianzas finales como los pollos de ceba y ponedoras comerciales (Espinoza-Canaza, 2019).

La crianza de reproductores debe garantizar huevos fértiles, para que, después del proceso de incubación, se obtengan pollitos de primera que puedan remplazar los animales de la etapa final. Esto implica que en las etapas intermedias el manejo de los huevos y animales debe ser esmerado, óptimo, pues sobre la fertilidad de los huevos actúa un gran número de factores que son manejados por el hombre, y que de ellos depende la eficiencia, rentabilidad, del proceso productivo, otro tanto ocurre con la incubación. Cuando estas dos etapas no son eficientes, rentables, provocan, por lo general, un aumento en el costo de las producciones finales, afectando la economía de estas etapas, por el contrario, que no se produzcan los huevos y carne que demanda el consumo humano (Rosas \& Lerdon, 2018).

Es reconocido que uno de los elementos en que los avicultores coinciden son los resultados que no sólo se logran con un buen trabajo en las etapas finales (ceba y ponedoras comerciales) que son fiables, hay que lograr reemplazos en cantidad y calidad que aseguren las necesidades de estas producciones terminales. Es por ello que, en la actualidad, gastan grandes recursos y esfuerzos en etapas intermedias, como son la incubación artificial y los reproductores, donde se obtienen los animales para las crianzas finales como los pollos de ceba y ponedoras comerciales (Acosta-Lozano et al. 2018). 
En razón de lo planteado, la investigación tiene por objetivo analizar los parámetros productivos en la incubación de huevos considerados como no aptos (por su peso y forma) procedentes de reproductoras pesadas.

\section{MATERIALES Y MÉTODOS}

La investigación se desarrolló, siguiendo los siguientes preceptos metodológicos, los cuales se describen a continuación:

\section{Lugar de investigación}

Se desarrolló en las granjas: "Amalia", "El Guayabal" y "Flor Canela", ubicadas en la parroquia Madre Tierra, cantón Mera, provincia de Pastaza y el proceso de incubación en la planta "INCUPASTAZA", ubicada en la vía Shell-Madre Tierra, km 4, parroquia Madre Tierra, cantón Mera, provincia de Pastaza. Con una duración del trabajo de campo de 60 días, distribuidos en el proceso de incubación, toma de datos y análisis de resultados.

\section{Muestra poblacional}

Se utilizaron1200 huevos divididos en cuatro grupos, los mismos que fueron: 300 huevos pequeños, 300 huevos grandes, 300 huevos deformes y 300 huevos normales, procedentes de reproductoras pesadas. El tamaño de la unidad experimental fue de 100 huevos. Cada tratamiento tuvo 3 repeticiones.

\section{Materiales, equipos e insumos}

Los materiales, equipos e insumos utilizados fueron los siguientes:

Materiales

1. Huevos "aptos" (normales).

2. Huevos "no aptos" (pequeños, grandes y deformes).

3. Flexómetro. 
4. Pie de rey.

5. Overol.

6. Guantes.

7. Cámara fotográfica.

8. Libreta de apuntes.

9. Materiales de oficina.

\section{Equipos}

1. Máquinas incubadoras.

2. Ovoscopio.

3. Pistolas de vacunación.

4. Campanas criadoras.

5. Balanza digital.

6. Computador personal.

Insumos

1. Desinfectante.

2. Amonio cuaternario, formol.

3. Biológicos: Mareck, Bronquitis.

\section{Tratamiento y diseño experimental}

Se evaluó el efecto de la incubación de huevos considerados como no aptos (pequeños, grandes y deformes) procedentes de reproductoras pesadas, para ser comparados con un grupo control (huevos aptos o normales), por lo que se contó con 4 tratamientos experimentales y cada uno con 3 repeticiones. Las unidades experimentales se distribuyeron bajo un Diseño Completos al Azar (DCA), y que para su análisis se ajustó al siguiente modelo lineal aditivo: Yij $=\mu+T i+\epsilon$ ij Dónde: Yij= Valor del parámetro en determinación $\mu=$ Media general $\mathrm{T}$ i = Efecto generado por tratamientos (tipo de huevos) $\epsilon \mathrm{ij}=$ Efecto del error experimental. 


\section{Mediciones experimentales}

Las mediciones experimentales consideradas fueron las siguientes:

1. Eclosionalidad, \%.

2. Incubabilidad, \%.

3. Viabilidad, \%.

4. Peso de los pollitos al nacimiento, g.

5. Mortalidad de los pollitos al primer día de nacidos

\section{Análisis estadísticos y pruebas de significancia}

Los resultados experimentales fueron sometidos a los siguientes análisis estadísticos:

1. Análisis de varianza para las diferencias (ADEVA). En el cuadro 1, se reporta el esquema del ADEVA empleado.

2. Separación de medias mediante la prueba de Tukeyal nivel de significancia $\mathrm{P} \leq 0,05$.

\section{Cuadro 1.}

Esquema del ADEVA empleado.

\begin{tabular}{|l|c|}
\hline \multicolumn{1}{|c|}{ Fuente de Variación } & Grados de Libertad \\
\hline Total & 11 \\
\hline Tratamientos & 3 \\
\hline Error & 8 \\
\hline
\end{tabular}

Elaboración: Autores (2021). 


\section{RESULTADOS Y DISCUSIÓN}

En este apartado se presentan los resultados de la investigación, con su respectivo análisis con la finalidad de cumplir con el objetivo de la investigación, en este sentido, se presenta:

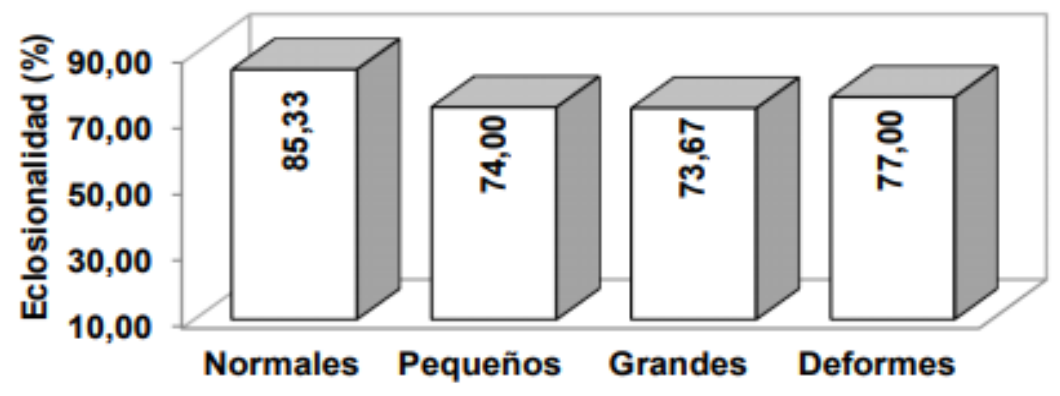

Tipo de Huevos

\section{Gráfico 1.}

Eclosionalidad de diferentes tipos de huevos de aves pesadas en el proceso de incubación.

Elaboración: Los autores.

El análisis de la eclosionalidad de los huevos normales fueron de $85,33 \%$, valor que difiere significativamente $(P<0,05)$ de los huevos pequeños y grandes puesto que se encontraron valores de 74,00 y $73,67 \%$ respectivamente, en cambio que comparten el rango de significancia con la respuesta de los huevos deformes en los que se determinaron un índice de eclosionalidad de $77.00 \%$ (Gráfico 1), respuestas que permiten establecer que el tamaño de los huevos influye en la característica eclosión o rotura de la cascara del huevo para que el pollito salga , puesto que las incubadoras están diseñadas para utilizar huevos medianos lo que hace que afecte la eclosión de huevos muy grandes, pequeños y en parte de los deformes. 
Revista Arbitrada Interdisciplinaria KOINONIA

Año VI. Vol VI. N 12 . Julio - Diciembre. 2021

Hecho el depósito de Ley: FA2016000010

ISSN: 2542-3088

FUNDACIÓN KOINONIA (F.K). Santa Ana de Coro. Venezuela.

Junior Stalin Vargas Hidalgo; Diego Armando Masaquiza-Moposita; Nelson Rene Ortiz Naveda

\section{Cuadro 2.}

Efecto del tamaño y forma de los huevos en el proceso de incubación.

Cuadro 6. EFECTO DEL TAMAÑO Y FORMA DE LOS HUEVOS EN EL PROCESO DE INCUBACIÓN.

\begin{tabular}{|c|c|c|c|c|c|c|c|c|c|}
\hline \multirow{3}{*}{$\begin{array}{l}\text { Variables } \\
\text { Eclosionalidad, \% }\end{array}$} & \multicolumn{7}{|c|}{ Tipo de huevos } & \multirow{2}{*}{ E.E. } & \multirow{3}{*}{$\begin{array}{l}\text { Prob. } \\
0.03\end{array}$} \\
\hline & \multicolumn{2}{|c|}{ Normales } & \multicolumn{2}{|c|}{ Pequeños } & \multicolumn{2}{|c|}{ Grandes } & Deformes & & \\
\hline & 85.33 & a & 74.00 & $\mathrm{~b}$ & 73.67 & $\mathrm{~b}$ & 77.00 & 2.43 & \\
\hline Incubabilidad, \% & 89.28 & a & 79.48 & a & 81.79 & a & 82.39 & 2.66 & 0.13 \\
\hline Viabilidad, \% & 91.05 & a & 83.19 & a & 83.68 & a & 84.60 & 2.25 & 0.12 \\
\hline Peso de los pollitos al nacimiento, $\mathrm{g}$ & 45.91 & c & 35.14 & d & 53.84 & a & 48.65 & 0.35 & 0.00 \\
\hline Mortalidad al día de nacidos, $\%$ & 0.00 & & 0.00 & & 0.00 & & 0.00 & & \\
\hline
\end{tabular}

E.E.: Error estándar.

Prob. > 0.05: No existen diferencias estadísticas (ns).

Prob. $<0.05$ : Existen diferencias significativas $\left({ }^{*}\right)$.

Prob. < 0.01: Existen diferencias altamente significativas $\left({ }^{\star *}\right)$.

Medias con letras iguales no difieren estadísticamente de acuerdo a la prueba de Tuckey.

Elaboración: Los autores.

Los porcentajes más altos de eclosionalidad, se obtuvieron en el grupo control (huevos normales), dichos porcentajes coincide con los reportados por (Donohue, 2010), quien encontró índices de eclosión en huevos procedentes de reproductoras pesadas de $84,5 \%$, en el resto de los tratamientos los valores fueron más bajos, (Medina, 2012), reporta índices de eclosión de $83,60 \%$ en aves pesadas.

Otros autores como (Sandoval et al. 2005), reportan un porcentaje de eclosión de 82,65\% de esta manera se puede manifestar que el tamaño y tipo de huevos que se utiliza en el proceso de incubación tiene que ver mucho en la eclosión, los mismos que representan la disponibilidad de la producción avícola en el sector agropecuario que garantiza la seguridad y soberanía alimentaria de la población ya sea como la disponibilidad de carne de ave (pollo). 


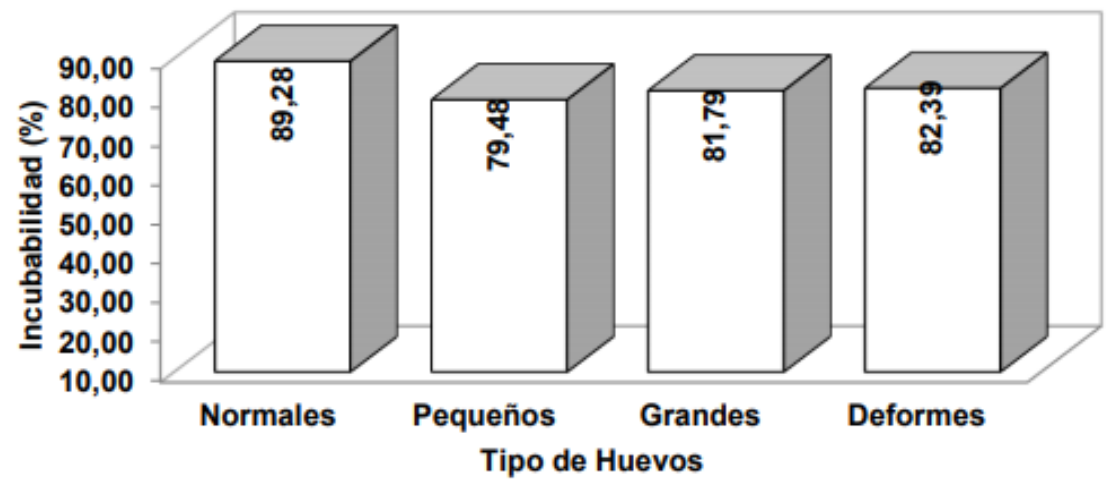

\section{Gráfico 2.}

Índice de incubabilidad de diferentes tipos de huevos de aves pesadas en el proceso de incubación.

Elaboración: Los autores.

Conociendo que la incubabilidad es la capacidad de un huevo fértil de producir un pollito vivo y apto, y se mide en porcentajes que están dados por los huevos fértiles cargados en la incubadora y los pollitos nacidos (Andrade, C. 2011); se determinó que los huevos normales, pequeños, grandes y deformes presentaron índices de incubabilidad de 89,28, $79,48,81,79$ y $82,39 \%$ respectivamente (Gráfico 2), valores entre los cuales no existen diferencias estadísticas $(P>0,05)$, por lo que se considera que el peso y la forma de los huevos no tienen incidencia en que de ellos se obtengan pollitos viables.

Se indica que en razas de ponedoras pesadas obtuvo índices de incubabilidad entre 72 y $73 \%$, en cambio los valores encontrados en los huevos normales, grandes y deformes coinciden con los informados por (Álvarez-Mira et al. 2017), quienes al estudiar este indicador en huevos procedentes de reproductoras pesadas obtuvieron valores del $85 \mathrm{al}$ $87 \%$, aunque en el caso de los huevos pequeños los valores hallados $(79.48 \%)$, son inferiores, lo cual pudiera establecer una relación entre el tamaño del huevo y la incubabilidad. 


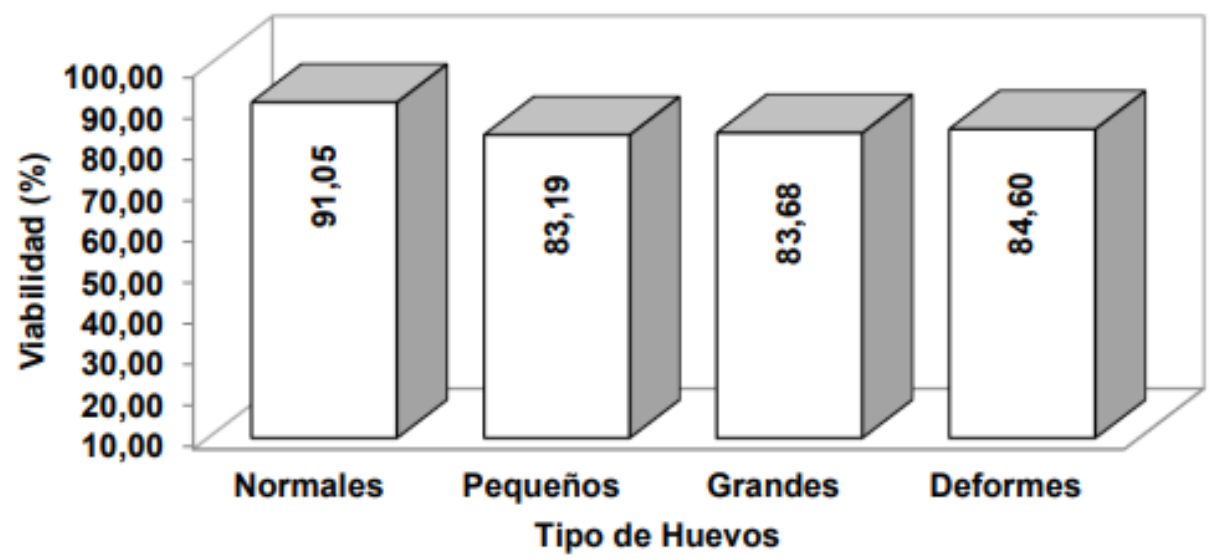

Gráfico 3. Viabilidad de diferentes tipos de huevos de aves pesadas en el proceso de incubación.

Elaboración: Los autores.

El porcentaje de viabilidad, representa la cantidad de pollitos nacidos vivos de los huevos que fueron establecidos como fértiles, en esta investigación se determinó que dicho porcentaje a pesar de presentar valores numéricamente diferentes entre los tipos de huevos evaluados, estadísticamente son iguales $(P>0.05)$, por cuanto al incubar huevos normales, pequeños, grandes y deformes, se encontraron índices de viabilidad de 91,05, $83,19,83,68$ y $84.60 \%$ respectivamente (Gráfico 3), respuestas que determinan que el peso y la forma de los huevos no influyen en el índice de viabilidad de los pollitos al nacimiento, sino que este dependerá de la calidad de los huevos, es decir de su fertilidad, que es la capacidad de un óvulo de ser fertilizado por un espermatozoide para producir un embrión.

Los valores encontrados en el presente estudio están por encima de los encontrados por (Álvarez-Mira et al. 2017), quien al evaluar este porcentaje en huevos procedentes de reproductoras pesadas observó que la viabilidad osciló entre el 82 y $87 \%$; siendo también superiores a los mencionados por (Cortes-Cuevas et al. 2016), quienes plantean que la viabilidad en ponedoras debe estar en un $80 \%$ para calificarla como buena. Sin embargo, De están manera se puede manifestar que la viabilidad de los huevos no está dada por el tipo de los huevos, sino por otros factores externos, ya que los huevos sean estos 
normales, grandes, pequeños o anormales estos son prácticamente viables, por lo que este factor posiblemente sea necesario únicamente para disponer de una parvada homogénea, particularidad que se debe tener en cuenta según el objetivo de la cría que se obtiene en los galpones de acabado de estas aves.

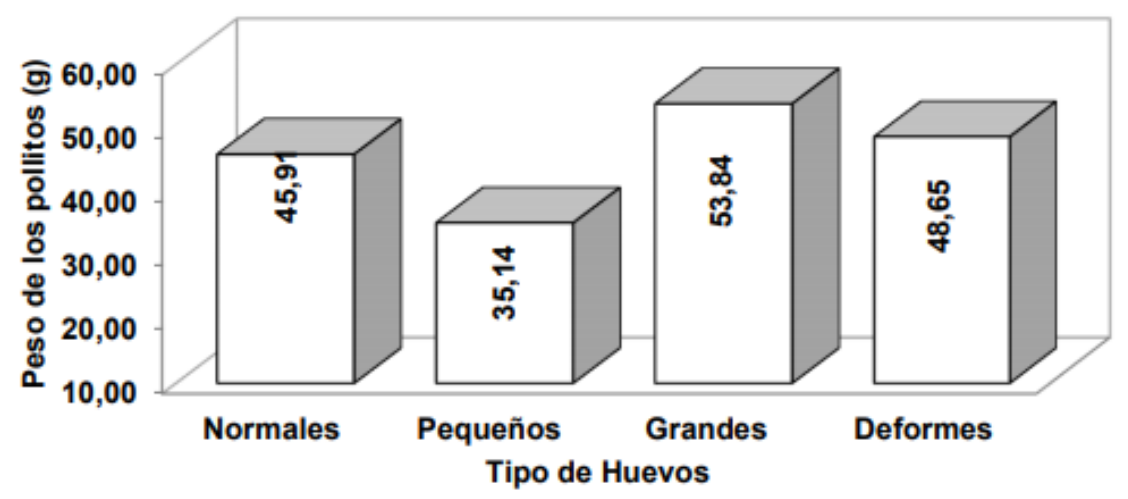

Gráfico 4. Peso (g) de los pollitos provenientes de diferentes tipos de huevos de aves pesadas en el proceso de incubación.

Elaboración: Los autores.

Los pesos obtenidos están por encima de los mencionados en varias investigaciones, entre las que se pueden citar las siguientes: Nápoles, M. (2000) reportó pesos de los pollitos al nacimiento de $34.6 \mathrm{~g}$ obtenidos de huevos considerados aptos y $34 \mathrm{~g}$ para los no aptos; (Guerra-Casas et al. 2019), refieren en trabajos realizados en huevos cuyos pesos eran de $49.65 \mathrm{~g}$, haber obtenido pollitos con un peso de $32.82 \mathrm{~g}$ al nacimiento; de igual manera, la industria acepta un peso mínimo del huevo, requerido para una incubación exitosa, de $52 \mathrm{~g}$; esto, con la finalidad de asegurar una buena calidad del pollo cuando el principal rasgo de calidad a evaluar sea el peso. La relación del peso del pollo y el peso del huevo se mantiene constante en la mayoría de las especies aviares. El peso del pollo esta normalmente en el rango del 62 al $76 \%$ del peso inicial del huevo.

Considerándose que las explotaciones avícolas consideran que pollitos de pesos menores a 40 gramos no son viables, se considera que es preferible incubar huevos 
considerados normales, grandes y deformes, porque de ellos se obtendrán pollitos con pesos superiores a los $40 \mathrm{~g}$, ya que además, los pollitos obtenidos de huevos pequeños al llegar a los $40 \mathrm{~g}$, se consideran como pollitos de segunda, los mismos que no cumplirían con las metas propuestas en las explotaciones avícolas, como es el de producir animales con mayores pesos en un menor tiempo.

Los pesos de los pollitos al nacimiento procedentes de huevos grandes fueron de 53,84 g, valores que difieren significativamente ( $P$ 45,91 y 35,14 g respectivamente (Gráfico 4). De esta manera se puede manifestar que el tamaño de los huevos es un factor decisivo que determinar el peso de los pollos al nacimiento.

\section{CONCLUSIONES}

Los porcentajes de eclosionalidad fue menor en los huevos grandes y pequeños (73.67 y $74.00 \%$, en su orden), es decir presentar mayor dificultad para romperse la cascara para liberar al pollito, no así en los huevos de tamaño normal que alcanzaron un índice de $85.33 \%$.

Respecto a los índices de incubabilidad estos variaron numéricamente entre 79.48 y $89.28 \%$, con mejores respuestas en los huevos normales, sin embargo, se encontró que el tamaño y forma de los huevos no influye en este parámetro.

50Los índices de viabilidad alcanzados fueron de 83.19 a $91.05 \%$, que pertenecen a los obtenidos en los huevos pequeños y normales, respectivamente, pero que estadísticamente son iguales.

Se encontró que el peso de los pollitos al nacimiento está en función del peso del huevo del que proceden, por cuanto los pollos que presentaron los mayores pesos al nacimiento $(53.84 \mathrm{~g})$ fueron los que provinieron de los huevos grandes, a diferencia de los pollitos de los huevos pequeños que presentaron los menores pesos (35.14 g).

También se determinó que no existió relación entre la deformación del huevo y la posibilidad de su utilización, por presentar resultados entre los anteriormente mencionados. 


\section{FINANCIAMIENTO}

No monetario.

\section{AGRADECIMIENTO}

A la Escuela Superior Politécnica de Chimborazo, Riobamba por el apoyo a la investigación.

\section{REFERENCIAS CONSULTADAS}

Acosta-Lozano, N., González Asencio, M., Duque Marín, R., \& Andrade Yucailla, V. (2018). Producción de pollos criollos con una incubadora artesanal de huevos en la comuna San Vicente cantón Santa Elena [Production of Creole chickens with an artisanal incubator of eggs in the San Vicente commune, Santa Elena canton]. Revista Científica Y Tecnológica UPSE, 5(1), 90-95. https://doi.org/10.26423/rctu.v5i1.336

Álvarez-Mira, D., Faracica Turizo, C., \& Hortúa López, L. (2017). Colibacilosis aviar en una granja de reproductoras pesadas en Cundinamarca [Avian colibacillosis in a broiler breeder farm in Cundinamarca]. Conexión Agropecuaria JDC, 7(1), 47-56.

Andrade, C. (2011). Determinación de Parámetros Reproductivosde gallinas criollas para huevos verdes desde la recolección de huevos hasta su etapa inicial. Tesis para obtener el título de ingeniero zootecnista. Escuela Superior Politécnica de Chimborazo. Andrade Galarza, C.S [Determination of Reproductive Parameters of Creole hens for green eggs from the collection of eggs to their initial stage. Thesis to obtain the title of zootechnical engineer. Chimborazo Higher Polytechnic School. Andrade Galarza, C.S]. http://dspace.espoch.edu.ec/handle/123456789/1816

Cortes-Cuevas, A, Cedillo Monrroy, M., Gómez Verduzco, G., Balderas González, A., \& Avila González, E. (2016). Producción y calidad del huevo en gallinas en semilibertad alimentadas con diferentes niveles de pasta de canola [Egg production and quality in semi-free-range hens fed different levels of canola meal]. Revista mexicana de ciencias pecuarias, 7(2), 173-184.

De Marchi, G., Chiozzi, G., \& Fasola, M. (2008). Solar Incubation Cuts down Parental Care in a Burrow Nesting Tropical Shorebird, the Crab Plover Dromas ardeola. Journal of Avian Biology, 39(5), 484-486. http://www.jstor.org/stable/30244474 
Donohue, M. (2010). 20 años de mejoramiento avícola: Pollo de Engorde [20 years of poultry improvement: Broiler Chicken]. https://n9.cl/8ah61

Espinoza-Canaza, F. (2019). Construcción y evaluación del funcionamiento de un prototipo de incubadora para usos múltiples, Chachapoyas, 2018 [Construction and evaluation of the operation of a prototype of an incubator for multiple uses, Chachapoyas, 2018]. Revista Científica UNTRM: Ciencias Naturales E Ingeniería, 2(1), 26 - 32. http://dx.doi.org/10.25127/ucni.v2i1.444

Guerra-Casas, L., Cabrera Morales, I., Aldana Herrera, Y., Collante Cánovas, M., \& Guevara Viera, R. (2019). Pérdidas económicas por huevos de reproductoras pesadas no aptos para la incubación (deformes, pequeños, grandes y rugosos) [Economic losses from broiler breeder eggs unfit for incubation (misshapen, small, large and rough)]. Revista De Producción Animal, 15(2).

López-Sobaler, A., Aparicio Vizuete, A., \& Ortega, M. (2017). Papel del huevo en la dieta de deportistas y personas físicamente activas [Role of the egg in the diet of athletes and physically active people]. Nutrición Hospitalaria, 34(Supl. 4), 3135. https://dx.doi.org/10.20960/nh.1568

Medina, J. (2012). Problemas del embrión de alta conformación y su incubación en sistemas de etapa única: manejo de incubadoras [Problems of the highconformation embryo and its incubation in single-stage systems: incubator management]. XXII Congreso Centroamericano y del Caribe de Avicultura en Panamá. Panamá. https://n9.cl/twr2

Mendoza-Rodríguez, Y., Brambila Paz, J., Arana Coronado, J., Sangerman-Jarquín, D., \& Molina Gómez, J. (2016). El mercado de huevo en México: tendencia hacia la diferenciación en su consumo [The egg market in Mexico: trend towards differentiation in its consumption]. Revista Mexicana de Ciencias Agrícolas, $7(6), 1455-1466$.

Rosas, N., \& Lerdon, F. (2018). Factibilidad de un proyecto avícola para producción de huevos bajo sistema free-range en el sur de Chile [Feasibility of a Project for poultry egg production system under Free-range in Southern Chile]. Idesia (Arica), 36(3), 131-140. https://dx.doi.org/10.4067/S0718-34292018005001702 
Sandoval, A., \& Yuño, M., \& Bakker, M.L, \& Rodríguez, E., \& Beretta, A. (2005). Aplicación de la embriodiagnosis para evaluar la eficiencia de la planta de incubación de barrilleros en una empresa avícola comercial en la Argentina [Application of embryodiagnosis to evaluate the efficiency of the barrel incubation plant in a commercial poultry company in Argentina]. RIA. Revista de Investigaciones Agropecuarias, 34(2),75-89.

(C2021 por los autores. Este artículo es de acceso abierto y distribuido según los términos y condiciones de la licencia Creative Commons Atribución-NoComercial-Compartirlgual 4.0 Internacional (CC BY-NC-SA 4.0) (https://creativecommons.org/licenses/by-nc-sa/4.0/). 